\title{
Robust Synchronization of the Unified Chaotic System
}

\author{
Hatem Trabelsi*, Mohamed Benrejeb* \\ * Laboratoire de Recherche en Automatique (LA.R.A), ENIT
}

\begin{tabular}{l} 
Article Info \\
\hline Article history: \\
Received Oct 6, 2014 \\
Revised Nov 31, 2014 \\
Accepted Dec 20, 2014 \\
\hline
\end{tabular}

Keyword:

Compound Matrices

Robustness

Synchronization

Unified Chaotic System

\begin{abstract}
This paper investigates the synchronization problem of the unified chaotic system. The case of identical, but unknown, master and slave unified chaotic systems is considered. Based on compound matrices formalism, a unified synchronization control scheme is proposed independently of the unknown system parameter. Simulation results are provided to show the effectiveness of the presented scheme.
\end{abstract}

Copyright (C) 2015 Institute of Advanced Engineering and Science. All rights reserved.

\section{Corresponding Author:}

Hatem Trabelsi, Laboratoire de Recherche en Automatique (LA.R.A), Ecole Nationale d'Ingénieurs de Tunis, BP 37, 1002 Tunis Belvédère, Tunisia

Email: 7atem.trabelsi@gmail.com

\section{INTRODUCTION}

Chaos synchronization is an attractive phenomenon involved in a variety of real-life processes. In 1990, Pecora and Carrol proved [1] that two chaotic systems can synchronize. This means that one system (slave system), can follow the trajectories of another one (master system), when an appropriate control law is applied. Since then, many synchronization schemes have been proposed [2], [3], [4], [5] such as nonlinear control [6], nonlinear observer [4], [7], [8] adaptive control [9], [10], [11] active control [12], [13], [14], fuzzy control [15], [16], and backstepping control [17], [18]. More recently, in 2002, Lü and Chen et al. [19] investigated some specific chaotic systems and described them in a unified form known as the unified chaotic system. This system plays a very important role in the study of the generalized Lorenz system family [20]. Different results related to the unified chaotic system are reported in literature [21], [22], [23], [24], [25].

In this paper, we propose a synchronization control scheme based on the concept of compound matrices, in order to synchronize two identical but unknown unified chaotic systems. Compound matrices [26], [27], have interesting spectral properties making of them a powerful tool for stability study [26], [28]. In [27], existence of Hopf Bifurcation in dynamical systems analysis and stability of matrices are investigated using the compound matrices formalism.

The paper is organized as follows. In Section 2, we introduce briefly the unified chaotic system and the theoretical tool used in this work, namely the compound matrix method. In Section 3, robust synchronization control scheme is proposed for identical but unknown master and slave unified chaotic systems. Obtained results are tested through numerical simulations, in Section 4.

\section{PROBLEM STATEMENT}

The unified chaotic system [19] can be expressed by the following differential equations: 


$$
\left\{\begin{array}{l}
\dot{x}_{1}=(25 \theta+10)\left(x_{2}-x_{1}\right) \\
\dot{x}_{2}=(28-35 \theta) x-x_{1} x_{3}+(29 \theta-1) x_{2} \\
\dot{x}_{3}=x_{1} x_{2}-(1 / 3)(8+\theta) x_{3}
\end{array}\right.
$$

where $x_{1}, x_{2}$ and $x_{3}$ are state variables and $\theta$ a constant parameter.

For $\theta$ varying continuously in [0,1], the whole family of systems is chaotic [29]. It includes, in particular, the canonical Lorenz [30], Chen [31] and Lü [29] chaotic systems respectively for $\theta=0,1$ and 0.8 .

Let system (1) be the master system and define the slave system as

$$
\left\{\begin{array}{l}
\dot{y}_{1}=(25 \theta+10)\left(y_{2}-y_{1}\right)+u_{1} \\
\dot{y}_{2}=(28-35 \theta) y-y_{1} y_{3}+(29 \theta-1) y_{2}+u_{2} \\
\dot{y}_{3}=y_{1} y_{2}-(1 / 3)(8+\theta) y_{3}+u_{3}
\end{array}\right.
$$

where $y_{1}, y_{2}$ and $y_{3}$ are state variables of the slave system, $\theta$ the parameter introduced for the master system. Given the error vector $e=\left(e_{1}, e_{2}, e_{3}\right)^{T}$ defined by

$$
e_{i}=y_{i}-x_{i}, i=1 . .3
$$

$u_{1}, u_{2}$ and $u_{3}$ are the control laws to be designed such that the following error dynamical system (4) is stable

$$
\left\{\begin{array}{l}
\dot{e}_{1}=(25 \theta+10)\left(y_{2}-y_{1}\right)-(25 \theta+10)\left(x_{2}-x_{1}\right)+u_{1} \\
\dot{e}_{2}=(28-35 \theta) y_{1}-y_{1} y_{3}+(29 \theta-1) y_{2}-(28-35 \theta) x_{1}+x_{1} x_{3}-(29 \theta-1) x_{2}+u_{2} \\
\dot{e}_{3}=y_{1} y_{2}-(1 / 3)(8+\theta) y_{3}-x_{1} x_{2}+(1 / 3)(8+\theta) x_{3}+u_{3}
\end{array}\right.
$$

Define the extended state vector $\varphi=\left(\varphi_{i}\right)$ as

$$
\varphi=\left(x_{1}, x_{2}, x_{3}, y_{1}, y_{2}, y_{3}\right)^{T}
$$

and the matrices $T$ and $N$ by

$$
\begin{aligned}
& T=\left(\begin{array}{cccccc}
-1 & 0 & 0 & 1 & 0 & 0 \\
0 & -1 & 0 & 0 & 1 & 0 \\
0 & 0 & -1 & 0 & 0 & 1
\end{array}\right) \\
& N(.)=\left(\begin{array}{cccccc}
25 \theta+10 & -25 \theta-10 & 0 & -25 \theta-10 & 25 \theta+10 & 0 \\
-28+35 \theta & -29 \theta+1 & x_{1} & 28-35 \theta & 29 \theta-1 & -y_{1} \\
0 & -x_{1} & \frac{8}{3}+\frac{1}{3} \theta & 0 & y_{1} & -\frac{8}{3}-\frac{1}{3} \theta
\end{array}\right)
\end{aligned}
$$

such that the error vector (3) and the dynamical error chaotic system (4) can be expressed by

$$
\begin{aligned}
& e=T \varphi \\
& \dot{e}=N(.) \varphi+u
\end{aligned}
$$

with

$$
u=K(.) \varphi
$$

and $K()=.\left(k_{i j}().\right) \in \mathbf{R}^{3 \times 6}$ is a non constant control gain matrix to be calculated such that (9) is stable. 
Assume, furthermore, that there exists a matrix $A()=.\left(a_{i j}().\right) \in \mathbf{R}^{3 \times 6}$ such that

$$
N(.)+K(.)=A(.) T
$$

Therefore, the dynamical error system (4) can be reduced to

$$
\dot{e}=A(.) e
$$

Our aim consists on expressing matrix $A($.$) entries, which depend on those of matrix K($.$) , then calculating the$ gain matrix $K($.$) such that system (12) is stable. In the sequel, we use, for simplicity, the notation k_{i j}$ instead of $k_{i j}($.$) .$

\section{PROPOSED ROBUST SYNCHRONIZATION SCHEME OF THE MASTER-SLAVE UNIFIED CHAOTIC SYSTEM}

\subsection{Basic Idea}

Synchronization between the master system (1) and the slave system (2) is equivalent to the stability of the dynamical error system (12). The stability study of the the characteristic matrix $A($.) of system (12) is performed based on the compound matrix method. Related preliminary notions are introduces in the following.

Let $M_{n}(\mathbf{R})$ be the linear space of matrices of size $n \times n$ with entries in $\mathbf{R}$ and let $A$ be a matrix in $M_{n}(\mathbf{R})$ and $k$ an integer in $[1, n]$. We note by $\wedge$ the exterior product in $\mathbf{R h}^{\mathfrak{h}}$.

Definition 1 [26], [27]: The additive compound matrix $A^{[k]}$ of $A$, with respect to the canonical basis in the $k^{\text {th }}$ exterior product space $\Lambda^{k} \mathbf{R}^{n}$ is a linear operator on $\Lambda^{k} \mathbf{R}^{n}$ and can be defined on a decomposable element $v_{1} \wedge v_{2} \wedge \ldots \wedge v_{k}$ by

$$
A^{[k]}\left(v_{1} \wedge \ldots \wedge v_{k}\right)=\sum_{i=1}^{k} v_{1} \wedge \ldots \wedge A v_{i} \wedge \ldots \wedge v_{k}, \forall v_{1} \wedge \ldots \wedge v_{k} \in \mathbf{R}^{n}
$$

Relations between entries $\left(a_{i j}\right)$ of $A$ and those of $A^{[k]}\left(\tilde{a}_{i j}\right)$ are linear.

Let $i$ be an integer in $\left[1, C_{n}^{k}\right]$. If we note by $(i)=\left(i_{1}, \ldots, i_{k}\right)$ the $i^{\text {th }}$ member in the lexicographic ordering of integer k-tuples such that $1 \leq i_{1}<\ldots<i_{k} \leq n$, we can obtain the additive compound matrix entries from the following result.

Proposition 1 [26], [27]:

$$
\tilde{a}_{i j}= \begin{cases}a_{i_{1} i_{1}}+\ldots+a_{i_{k} i_{k}}, & \text { if }(i)=(j), \\ (-1)^{r+s} a_{j_{r} i_{s}}, & \text { if exactly one entry } i_{s} \text { of }(i) \text { does not occur in }(j) \\ & \text { and } j_{r} \text { does not occur in }(i), \\ 0 & \text { if }(i) \text { differs from }(j) \text { in two or more entries. }\end{cases}
$$

In particular, we have $A^{[1]}=A, A^{[n]}=\operatorname{trace}(A)$ and for $A \in M_{3}(\mathbf{R})$

$$
A^{[2]}=\left(\begin{array}{ccc}
a_{11}+a_{22} & a_{23} & -a_{13} \\
a_{32} & a_{11}+a_{33} & a_{12} \\
-a_{31} & a_{21} & a_{22}+a_{33}
\end{array}\right)
$$

Definition 2 [27]: Let $|$.$| a vector norm on M_{n}(\mathbf{R})$ and $A$ a matrix in $M_{n}(\mathbf{R})$. 
The Lozinskiǐ measure (logarithmic measure) $\mu$ of $A$ with respect to $|$.$| is defined by$

$$
\mu(A)=\lim _{h \rightarrow 0^{+}} \frac{|I+h A|-1}{h}
$$

As examples, Lozinskiǐ measure of a matrix $A$ with respect to the three common vector norms $|x|_{1}=\sum_{i}\left|x_{i}\right|,|x|_{2}=\sqrt{\sum_{i}\left|x_{i}\right|^{2}}$ and $|x|_{\infty}=\sup _{i}\left|x_{i}\right|$ are

$$
\mu_{1}(A)=\sup _{j}\left(a_{j j}+\sum_{i, i \neq j}\left|a_{i j}\right|\right), \mu_{2}(A)=s\left(\frac{A+A^{T}}{2}\right) \text { and } \mu_{\infty}(A)=\sup _{i}\left(a_{i i}+\sum_{j, j \neq i}\left|a_{i j}\right|\right)
$$

where $s(A)$ denotes the maximum real part of the eigenvalues of $A$.

Compound matrices present a powerful tool for the stability study of matrices. The following result will be us ed in the sequel.

Theorem 1 [27]: if $(-1)^{n} \operatorname{det}(A)>0$ then $A$ is stable if and only if there exists a Lozinskiǐ measure $\mu$ on $M_{m}(\mathbf{R})$ such that $\mu\left(A^{[2]}\right)<0, m=C_{n}^{2}$.

According to theorem 1, the stability of the characteristic matrix $A($.$) of system (12) can be studied through$ its determinant and its second compound matrix.

\subsection{Dynamical Error System Stability Study Based on Compound Matrices}

By solving equation (11), we obtain the characteristic matrix $A($.$) of the dynamical error system$

$$
A(.)=\left(\begin{array}{ccc}
-25 \theta-10-k_{11} & 25 \theta+10-k_{12} & -k_{13} \\
28-35 \theta-k_{21} & 29 \theta-1-k_{22} & -x_{1}-k_{23} \\
-k_{31} & x_{1}-k_{32} & -\frac{8}{3}-\frac{1}{3} \theta-k_{33}
\end{array}\right)
$$

from which is deduced the second compound matrix as expressed in (14)

$$
A^{[2]}(.)=\left(\begin{array}{ccc}
4 \theta-11-k_{11}-k_{22} & -x_{1}-k_{23} & k_{13} \\
x_{1}-k_{32} & -\frac{76}{3} \theta-\frac{38}{3}-k_{11}-k_{33} & 25 \theta+10-k_{12} \\
k_{31} & 28-35 \theta-k_{21} & \frac{86}{3} \theta-\frac{11}{3}-k_{22}-k_{33}
\end{array}\right)
$$

In addition, we obtain relations between entries of matrix $K($.$) which is a block interdependent matrix$

$$
\left\{\begin{array}{lll}
k_{14}=-k_{11} & k_{15}=-k_{12} & k_{16}=-k_{13} \\
k_{24}=-k_{21} & k_{25}=-k_{22} & k_{26}=y_{1}-x_{1}-k_{23} \\
k_{34}=-k_{31} & k_{35}=x_{1}-y_{1}-k_{32} & k_{36}=-k_{33}
\end{array}\right.
$$

Referring to the compound matrix entries and the determinant of matrix $A($.$) , we propose, by the use of$ theorem 1, the following results.

Theorem 2: Global synchronization is achieved between unified chaotic systems described by (1) and (2) independently of the parameter $\theta$, if the following control law is applied 


$$
\left\{\begin{array}{l}
u_{1}=\left(\alpha+\left|x_{1}\right|\right)\left(x_{1}-y_{1}\right) \\
u_{2}=\left(1.16 \alpha-2.28+1.16\left|x_{1}\right|+\beta\right)\left(x_{2}-y_{2}\right)+\left(y_{1}-x_{1}\right) y_{3} \\
u_{3}=\left(x_{1}-y_{1}\right) y_{2}
\end{array}\right.
$$

where $\alpha>15.33$ and $\beta>44.50$.

Proof:

All diagonal elements of the compound matrix $A^{[2]}$ depend on $k_{11}$ and $k_{22}$. Let look for a gain matrix involving only $k_{11}$ and $k_{22}$ and consequently $k_{14}$ and $k_{25}$. By substituting all other $k_{i j}$ elements in $A$ by 0 , matrices $A$ and $A^{[2]}$ become

$$
\begin{aligned}
& A(.)=\left(\begin{array}{ccc}
-25 \theta-10-k_{11} & 25 \theta+10 & 0 \\
28-35 \theta & 29 \theta-1-k_{22} & -x_{1} \\
0 & x_{1} & -\frac{8}{3}-\frac{1}{3} \theta
\end{array}\right) \\
& A^{[2]}(.)=\left(\begin{array}{ccc}
4 \theta-11-k_{11}-k_{22} & -x_{1} \\
x_{1} & -\frac{76}{3} \theta-\frac{38}{3}-k_{11} & 25 \theta+10 \\
0 & 28-35 \theta & \frac{86}{3} \theta-\frac{11}{3}-k_{22}
\end{array}\right)
\end{aligned}
$$

The determinant of matrix $A($.$) is given by$

$$
\begin{aligned}
\operatorname{det}(A(.))= & -50 \theta^{3}+\left(\frac{29}{3} k_{11}-\frac{25}{3} k_{22}-195\right) \theta^{2}+ \\
& \left(-25 x_{1}^{2}+1730-\frac{1}{3} k_{11} k_{22}+77 k_{11}-70 k_{22}\right) \theta \\
& -10 x_{1}^{2}-k_{11} x_{1}^{2}+720-\frac{80}{3} k_{22}-\frac{8}{3} k_{11} k_{22}-\frac{8}{3} k_{11}
\end{aligned}
$$

By applying theorem 1, using the Lozinskir measure with respect to $|\cdot|_{1}$, system (11) is stable if the following inqualities are satisfied

$$
\begin{aligned}
& 4 \theta-11-k_{11}-k_{22}+\left|x_{1}\right|<0 \\
& \left|x_{1}\right|-\frac{76}{3} \theta-\frac{38}{3}-k_{11}+|-28+35 \theta|<0 \\
& -\frac{11}{3}+|25 \theta+10|+\frac{86}{3} \theta-k_{22}<0 \\
& -50 \theta^{3}+\left(\frac{29}{3} k_{11}-\frac{25}{3} k_{22}-195\right) \theta^{2} \\
& +\left(\left(-25 x_{1}^{2}+1730-\frac{1}{3}\right) k_{11} k_{22}+77 k_{11}-70 k_{22}\right) \theta \\
& -10 x_{1}^{2}-k_{11} x_{1}^{2}+720-\frac{80}{3} k_{22}-\frac{8}{3} k_{11} k_{22}-\frac{8}{3} k_{11}<0
\end{aligned}
$$

Inequalities (25a), (25b) and (25c) are sufficient conditions guarantying that and (25d) is related to the determinant of matrix $A($.

Left-hand sides of inequalities (25a), (25b) and (25c), can be majorated given that $0 \leq \theta \leq 1$. Furthermore, polynomial inequality $(25 \mathrm{~d})$ can be satisfied when all monomials are non positive. 
Conditions (25a), (25b), (25c) and (25d) can be therefore reduced to

$$
\left\{\begin{array}{l}
k_{11}>46 / 3+\left|x_{1}\right| \\
k_{22}>60 \\
29 k_{11}-25 k_{22}<57
\end{array}\right.
$$

The gain matrix entry $k_{11}$ can be chosen in the form

$$
k_{11}=\left|x_{1}\right|+\alpha \text { with } \alpha>\frac{46}{3}=15.33
$$

Substituting (26c) in (27), it comes

$$
k_{22}>\frac{29}{25}\left|x_{1}\right|+\frac{29}{25} \alpha-\frac{57}{25}=1.16\left|x_{1}\right|+1.16 \alpha-2.28
$$

and a possible choice of the gain matrix entry $k_{22}$ is

$$
k_{22}=1.16\left|x_{1}\right|+1.16 \alpha-2.28+\beta \text { with } \beta>0
$$

Given the constraint on the parameter $\alpha$ and the new expression of $k_{22}$, (26c) holds for every $\beta>44.50$. Finally, by calculating the other entries of the gain matrix $K($.$) , according to (20), and using the relation the$ control law expression of theorem 2 is retrieved.

$$
u_{i}=\sum_{j=1}^{6} k_{i j} \varphi_{j}
$$

Note that $\alpha$ and $\beta$ represent tuning parameters for the designed controller used to enhance system performances. An optimal choice of these parameters is done through trial and error process.

\section{SIMULATION RESULTS}

In this section, 3 cases are considered to show the effectiveness of the proposed method: $\theta=0$ (Lorenz chaotic system), $\theta=0.8$ (Lü chaotic system) and $\theta=1$ (Chen chaotic system). Corresponding simulation results are represented respectively in figure 1, 2 and 3.
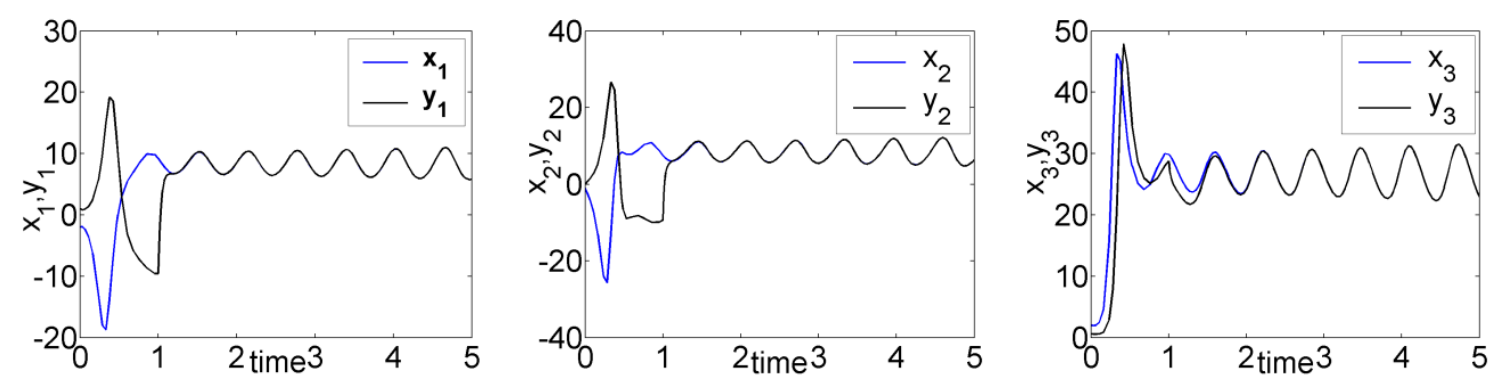

Figure 1. State trajectories of master and slave systems for $\theta=0$. Control is activated at time $\mathrm{t}=1$.

Differential equations are solved using the fourth-order Runge-Kutta method with a time step size equal to 0.001 . 

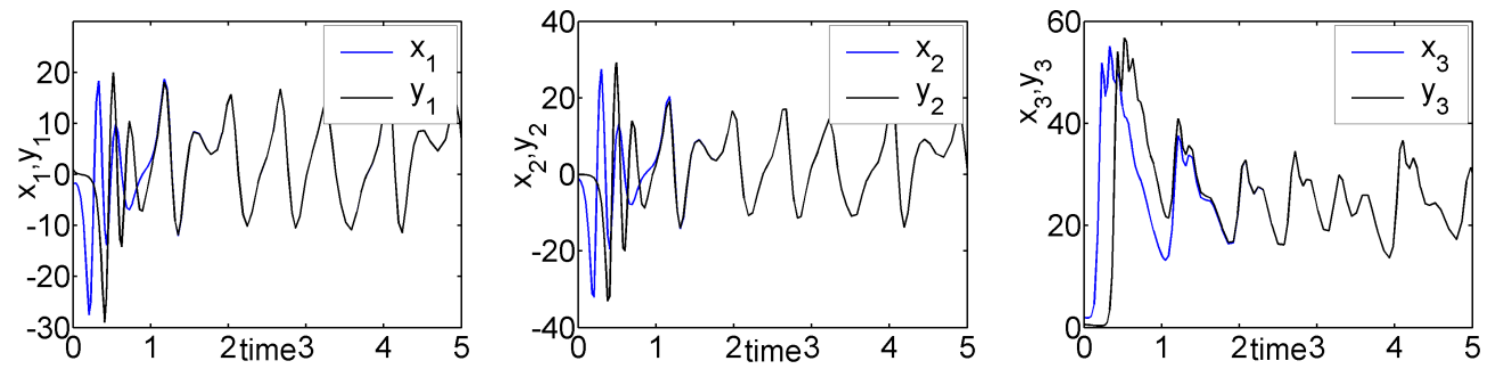

Figure 2. State trajectories of master and slave systems for $\theta=0.8$. Control is activated at time $\mathrm{t}=1$.

In all simulations, the constant parameters $\alpha$ and $\beta$ are respectively chosen equal to 15.5 and 55 . Different initial conditions are considered for the master and the slave systems and are respectively fixed to $(-2,-1,2)$ and $(1,0,0.6)$. In the three cases, we can notice that the trajectories of the controlled slave system synchronize with those of the master system. Numerical simulations have shown the effectiveness of the proposed method.
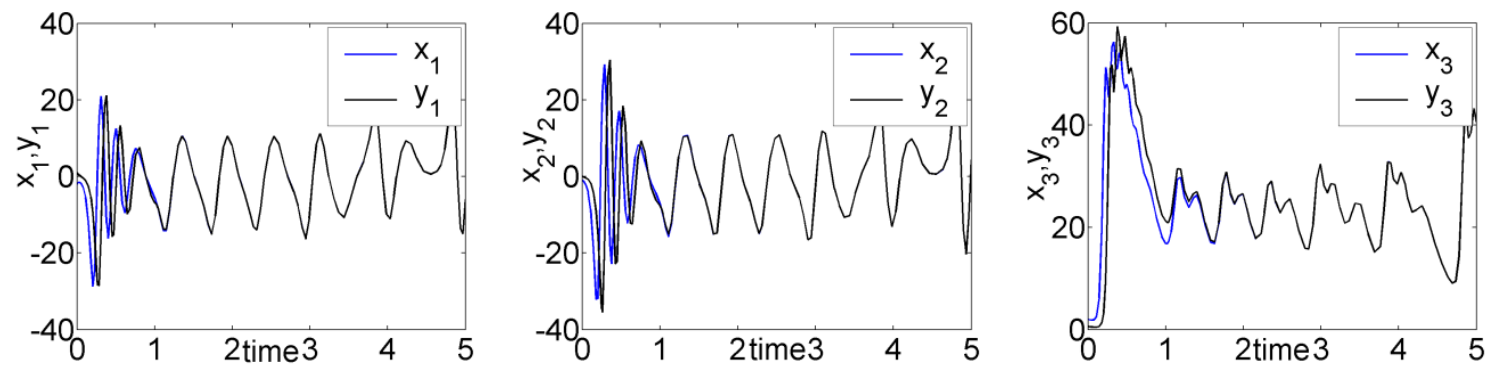

Figure 3. State trajectories of master and slave systems for $\theta=1$. Control is activated at time $\mathrm{t}=1$.

Unlike other reported results, as in [24], [32] and [33], the control law designed in this work is independent of the chaotic system parameter $\theta$. For this reason it's qualified as robust. Moreover, for the specific cases of Lorenz, Chen and Lü chaotic systems, the performed simulations indicate that synchronization is achieved faster than in other previous works [23], [24], [32].

\section{CONCLUSION}

In this paper, is investigated the synchronization of identical, but unknown, master and slave unified chaotic systems. The proposed synchronization scheme is based on compound matrices formalism. The obtained control law is independent of the unknown system parameter and is consequently efficient for all the family of considered chaotic systems. Numerical simulations are provided to illustrate the capability of the proposed method which can be applied to a large class of chaotic systems, with or without uncertainties. A possible extension of this work is the synchronization of two different unknown unified chaotic systems.

\section{REFERENCES}

[1] L.M. Pecora and T.L. Carroll, "Synchronization in chaotic systems". Phys. Rev. Lett. 64, pp. 821-825, 1990.

[2] L.M. Pecora and T.L. Carroll, “ Driving systems with chaotic signals”. Phys. Review, A 44 (4), pp. 2374-2383, 1991.

[3] E. Ott, C. Grebogy, and J.A. Yorke, "Controlling chaos”, Phys. Rev. Lett., 64 (11), pp. 1196-1199, 1990.

[4] H. Nijmeijer and I.M.Y. Mareels, "An observer looks at synchronization”, IEEE Trans. Cicuits Syst, 44, pp. 882890, 1997.

[5] P.P. Singh and H. Handa, "Various Synchronization Schemes for Chaotic Dynamical Systems", International Journal of Scientific Engineering and Technology, Volume No.1, Issue No.3, pp. 29-33, 2012. 
[6] E. Elabbasy, H. Agiza and M. El-Dessoky, "Global chaos synchronization for four scroll attractor by nonlinear control”, Sci. Res, Essay 1, pp. 65-71, 2006.

[7] J. Mata-Machuca, R. Martínez-Guerra and R. Aguilar-López, "An exponential polynomial observer for synchronization of chaotic systems", Commun. Nonlinear Sci. Numer. Simul., 15, pp. 4114-4130, 2010.

[8] Z. Zhang, H. Shao, Z. Wang and H. Shen, "Reduced-order observer design for the synchronization of the generalized Lorenz chaotic systems”, Appl. Math. Comput., 218, pp. 7614-7621, 2012.

[9] Y. Yu, "Adaptive synchronization of a unified chaotic system", Chaos, Solitons and Fractals, 36, pp. 329-333, 2008.

[10] M. Yan, X. Zheng and J. Zhen, "Synchronization of Hyperchaotic Systems under Active Adaptive Sliding Mode Control", TELKOMNIKA Indonesian Journal of Electrical Engineering, vol.11, no.11, pp. 6728-6736, 2013.

[11] E.A. Umoh, "Adaptive Hybrid Synchronization of Lorenz-84 System with Uncertain Parameters", TELKOMNIKA Indonesian Journal of Electrical Engineering, vol.12, no.7, pp. 5251-5260, 2014.

[12] E.W. Bai and K.E. Lonngren, "Synchronization of two Lorenz systems using active control", Chaos, Solitons and Fractals, 8, pp. 51-58, 1997.

[13] E.W. Bai and K.E. Lonngren, "Sequential synchronization of two Lorenz systems using active control", Chaos, Solitons and Fractals, 11, pp. 1041-1044, 2000.

[14] M. Benrejeb and S. Hammami, "New approach of stabilization of nonlinear continuous monovariable processes characterized by an arrow from matrix" 1ere Conférence Internationale Systems Eng. Design and Appl., SENDA, Monastir, 2008.

[15] V. Vembarasan and P. Balasubramaniam, "Chaotic synchronization of Rikitake system based on T-S fuzzy control techniques", Nonlinear Dyn, 74, pp. 31-44, 2013.

[16] H.T. Yau and C.S. Shieh, "Chaos synchronization using fuzzy logic controller", Nonlinear Analysis: Real World Applications, pp. 05-09, 2013.

[17] X. Tan X, J. Zhang and Y. Yang, "Synchronizing chaotic systems using backstepping design”, Chaos, Solitons and Fractals, 16, pp. 37-45, 2003.

[18] Y. Yu and S. Zhang, "Adaptive backstepping synchronization of uncertain chaotic systems”, Chaos, Solitons and Fractals, 21, pp. 643-649, 2004.

[19] J. Lu", G. Chen, D.Z. Cheng and S. Celikovsky, "Bridge the gap between the Lorenz system and Chen system", Int. J. Bifurcat. Chaos, 12, pp. 2917-2926, 2002.

[20] C. Tao, H. Xiong and F. Hu, "Two novel synchronization criterions for a unified chaotic system", Chaos, Solitons and Fractals, 27, pp. 115-120, 2006.

[21] J. Lu, X. Wu, X. Han and J. Lu“, “Adaptive feedback synchronization of a unified chaotic system”, Physics Letters, A, 329, pp. 327-333, 2004

[22] J. H. Park, "Stability criterion for synchronization of linearly coupled unified chaotic systems", Chaos, Solitons and Fractals, 23, pp. 1319-1325, 2005.

[23] Q. Zhang, S. Chen, Y. Hu and C. Wang, "Synchronizing the noise-perturbed unified chaotic system by sliding mode control”, Physica, A 371, pp. 317-324, 2006.

[24] F. Wang and C. Liu, "Synchronization of unified chaotic system based on passive control", Physica, D, 225, pp. 55-60, 2007.

[25] H. Wang, Z.Z. Han, W. Zhang and Q.Y. Xie, "Synchronization of unified chaotic systems with uncertain parameters based on the CLF", Nonlinear Analysis: Real World Applications, 10, pp. 715-722, 2009.

[26] M. Fiedler, "Additive compound matrices and an inequality for eigenvalues of symmetric stochastic matrices", Czechoslovak Mathematical Journal, vol. 24, no. 99, pp. 392-402, 1974.

[27] M.Y. Li and L. Wang, "A Criterion for Stability of Matrices", J. Math. Anal. App. 225, pp. 249-264, 1998.

[28] J.S. Muldowney, "Compound matrices and ordinary differential equations", The Rocky Mountain Journal of Mathematics, vol. 20, no. 4, pp. 857-872, 1990.

[29] J. Lu" and G. Chen, "A new chaotic attractor coined”, Int. J. Bifurcat. Chaos, 12 (3), pp. 59-661, 2002.

[30] E.N. Lorenz, "Deterministic non-periodic flows", J. Atmos. Sci., 20, pp. 130-41, 1963.

[31] G. Chen and T. Ueta, "Yet another chaotic attractor", Int. J. Bifurcat. Chaos, 9, pp. 1465-1466, 1999.

[32] W. Xing-yuan and S. Jun-mei, "synchronization of the unified chaotic system", Nonlinear Analysis, 69, PP. 34093416, 2008.

[33] S. Kuntanapreeda, "Chaos synchronization of unified chaotic systems via LMI", Physics Letters A 373, PP. 2837 $2840,2009$.

\section{BIOGRAPHIES OF AUTHORS}

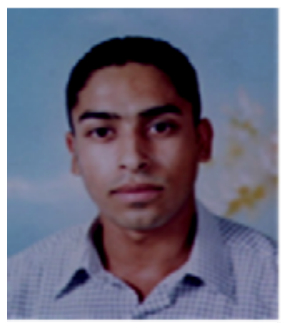

Hatem Trabelsi was born in Tunisia in 1980. He obtained the Master of Automatic Control and Signal Processing from the "Ecole Nationale d'Ingnieurs de Tunis" in 2007. He is currently a $\mathrm{PhD}$ student in Automatic Control and Signal Processing in LARA ENIT. His current research interests are control and synchronization of continuous and discrete chaotic systems. 


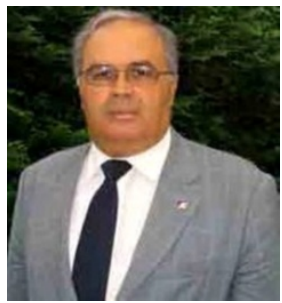

Mohamed BENREJEB has obtained his Diploma of "Ingénieur IDN" (French "Grande Ecole") in 1973, a Master degree of Automatic Control in 1974, a Ph.D. in Automatic Control of the University of Lille in 1976 and the DSc of the same University in 1980. He is currently a full professor at the "Ecole Nationale d'Ingénieurs de Tunis" and an invited Professor at the "Ecole Centrale de Lille". His research interests are in the area of analysis and synthesis of complex systems based on classical and non conventional approaches and recently in discrete event systems domain. 